20) Johnson W.C and Meyer J.R; Am. J. Obst. \& Gynce $9 ; 151,1925$

21) Helwig. F.C; Am. J. Obst. \& Gynec 26 ; 849. 1933

22) Steinharter R.H ; Beitr. z. path Anat. inz. 2lig. path 99. 148. 1937

23) Macgregor A.R ; Arch. Dis. Childhood. 14 ; 323. 1939

24) Benner M.C ; Arch. path 29; 4551940

25) Thaysen; Jahrbuch f. Kinderheilk. Bd. 79. 1401914

26) Browne; Brit. med. Journ N. 3195. 4691922

27) Kaldor; Am. Jcurn of Obst \& Gynec 25; 113. 1933

28) Ebergenyi ; Geburtsh. u. Frauenheilk. 2; 207, 1940

29）上野弘 未發表

30) Bruns \& Shields; Am. J. Obst \& Gynec. Vol. 61, Nr 5, 953. 1951

31) Küttner ; Virchows. Archiv Bd. 66. 1876

32) Stöhr; Lehrbuch d. Histolog. 1915

33) Tamassia ŭ Mirto; Ref, V. Olbrycht im Deutsche Zeitschr. f. d. gesamte gerichtl. Med. 9; 535, 1928.

34) Marx; Viertel Jahrsschr. f. gerichtl. Med. $54 ; 9,1917$.

35) Stieda; Zeitschr. f. wiss Zoolog. Bd 30 supplement 1878

36) Schulze; Die Lungen in Strickers Handbuch d. Lehre Von den Gewebe 1871.

37) Ebner ; Köllikers Handbuch d. Gewebelehre d. Menschen 6 auf. 1902.

38) Croix; Arch. f. Mik. Anat. Bd 22. 1863

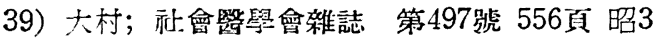

40) Emmert; Lehrbuch d. gerichtl. Med. S 363. 1900

41) Nippe; Zeitschr. f. Medizinalbeamte Bd. 41. Nr 20. ` 4731928.
42) Schönberg; Schweizerische Wochenschr. Jg $4 \mathrm{Nr} 14 \mathrm{~s} 351.1923$.

43) Feuger; Deutsche Zeitschr. f. d. gesamte gerichte. Med. Bd 11. s 275. 1923.

44) Beöthy; Zeitschr. f. Medizinal beamte Bd 41. s 379.1928

45) G. Stroßmann; Deutsche Med. Wocheuschr. Jg 46. s 385. 1920

46) Olbrycht ; Deutschr. f. d. gesamte gericht1. Med. Bd. 6. s 642. 1926

47) Fraenkel ŭ Weimann; Deutsche Zeitschr. f. d. gesammte gerichtl. Mєd. Bd 6. s 642 . 1926

48) Ungar; Viertel Jahrsschr. f. gerichtl. Med. Bd $45 ; 1$. supp-Heft. so 101. 1913.

49) Haberda; Beitr. f. gerichti. Med. Bd 1. 1911

50) Hocheim; Berliner klin. Wochenschr. H 21. s 542. 1915

51) Nippe; Vierteljahrsschr. f. gєricht1. Med. Bd 47. suppl-Heft s 641914

52) W. Weimann; Deutsche Zeitschr. f. d. gesamte gerichtl. Med. Bd 12. s 309. 1928.

53) Barthazard ŭ Lebrun; Ann d'hygiene qu. blique Bd 5. s 289. 1906 (Ref. V. Ruho im Vierteljahrsschr. f. gerichtl. Med. Bd 36. s 251. 1908)

54) Leers; Vierteljahrsschr. f. gericht1. Med. Bd 35. suppl-Heft s 31. 1908

55) Puppe; Vierteljahrsschr. f. gerichtl. Med. Bd 35. suppl-Heft s 33. 1908

56) Marx; Vierteljahrsschr. f. gerichtl. MedBd 54. s 9. 1917

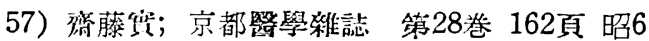

58) Meixner; Zieglers Beiträge Bd 37. s 502. 1905

59) Czerny; Zieglers Beiträge Bd 36. s 273. 1904

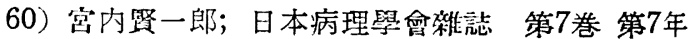

61）三谷 茂; 日本婦人科學會雜誌 策 30 巻 策 10,11 12 號 昭 10

\title{
誤植訂 正
}

\section{日醫大誌第 20 卷 1 號}

65 頁の表中右端五桁目 16 を 11 亿

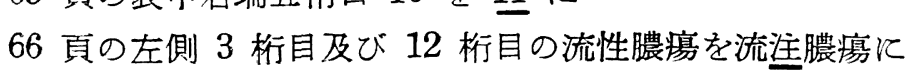

